\title{
Strain control of magnetocrystalline anisotropy and energy product of MnGa alloys
}

\author{
Nabil Al-Aqtash* and Renat Sabirianov \\ Department of Physics, University of Nebraska at Omaha, 6001 Dodge St., Omaha, NE, USA
}

We investigate the energy product of MnGa alloys as function of Mn concentration and applied elastic strain. Using density functional theory (DFT) based method we calculated the magnetocrystalline anisotropy (MAE) and magnetization of Mn-Ga alloys as function of composition, e.g. $\mathrm{Mn}$ and $\mathrm{Ga}$, and examined their variation under applied strain. Our calculations show that MAE is very large $\sim 22-27 \mathrm{Merg} / \mathrm{cm}^{3}$ in all three considered compositions, e.g. MnGa, $\mathrm{Mn}_{3} \mathrm{Ga}$ and $\mathrm{Mn}_{1.66} \mathrm{Ga}$. We show that MAE is very robust in MnGa system and remains large in wide range of concentrations and strains both compressive and tensile. We find that bi-axial tensile strain increases MAE in $\mathrm{Mn}_{1.66} \mathrm{Ga}$ alloys. Our study shows that the variation of MAE as function of Mn content is related to the change in electronic structure and, specifically, the Fermi level position with electron population variation. We estimated the theoretical limit of the energy product $(\mathrm{BH})_{\max }$ of $\mathrm{MnGa}, \mathrm{Mn}_{3} \mathrm{Ga}$ and $\mathrm{Mn}_{1.66} \mathrm{Ga}$ alloys as 23.65, 4.06 and 13.64 MGOe, respectively. We find that volume expansion of the MnGa alloys (by appropriate doping) should increase the magnetization and the energy product of these alloys.

* Corresponding author e-mail address: nalaqtash@unomaha.edu (N. Al-aqtash). 


\section{Introduction:}

High coercivity magnetic materials exhibiting a perpendicular magnetic anisotropy (PMA) and large magnetic energy product have great application potential in ultrahigh-density perpendicular magnetic recording media, high-performance permanent magnets and spintronic applications such as magnetic tunnel junctions (MTJs) and spin injectors [1-12]. Materials with large PMA possess interesting physical phenomena like efficient spin injection into semiconductors from ferromagnetic contact, giant anomalous Hall Effect, and long-lived ultrafast spin procession [13-15]. It is important to explore methods to control the magnetic anisotropy in crystalline thin films to develop functional and highly reliable devices $[10,16]$.

Mn-Ga alloys are PMA materials that have the thermodynamic stability, relatively large spin polarization, low saturation magnetization, square-like magnetization hysteresis loops, and perpendicular anisotropy [17-21]. Mn-Ga binary alloys show strong magnetism and large uniaxial magnetic anisotropy even though these alloys do not contain any noble, rare-earth metals or magnetic elements. $\mathrm{Mn}_{3} \mathrm{Ga}$ crystallizes in the $\mathrm{D} 0_{22}$ structure exhibits a unique combination of low magnetization, high uniaxial anisotropy, high Curie temperature and high spin polarization, which suit the requirements for spin torque memories down to $10 \mathrm{~nm}$ in size $[19,22]$. The structural, magnetic, and transport properties for off-stoichiometric $L 1_{0} \mathrm{Mn}-\mathrm{Ga}$ films have been investigated in view of their magneto-optical and spintronics applications [12,14,17,18]. $L 1_{0^{-}} \mathrm{Mn}_{50} \mathrm{Ga}_{50}$ homogeneous films were theoretically predicted to have large magnetocrystalline anisotropy (MAE) of $26 \mathrm{Merg} \cdot \mathrm{cm}^{-3}$, moderate magnetization of $2.51 \mu_{\mathrm{B}} / \mathrm{Mn}$, and large theoretical limit of magnetic energy product of 28.2 MGOe [20,23-25].

Alloys of $\mathrm{Mn}_{x} \mathrm{Ga}_{1-x}$ with $L 1_{0}$ structure are thermodynamically stable for $(x=0.5-0.65)$ with large MAE [26]. $\mathrm{DO}_{22}$ structures of $\mathrm{Mn}_{x} \mathrm{Ga}_{1-x}$ can be stabilized using certain preparation 
procedures at $x=0.5-0.65$. It exhibits strong ferrimagnetism, as confirmed by neutron scattering [27]. A high Curie temperature of up to $\sim 800 \mathrm{~K}$, large magnetic anisotropy, and compositionsensitive magnetization were also reported in $\mathrm{DO}_{22}$-phase polycrystalline bulk samples [28]. Furthermore, $\mathrm{Mn}_{x} \mathrm{Ga}(1<x<1.8)$ alloys with $L 1_{0}$ structures have increasing attention because of potential applications in ultrahigh-density magnetic recording media, permanent magnets and spintronics. Recently, $L 1_{0^{-}} \mathrm{Mn}_{1.5} \mathrm{Ga}$ epitaxial films grown on GaAs (001) have tunable perpendicular coercive field $(\mathrm{Hc})$ from 8.1 to $42.8 \mathrm{kOe}$, MAE up to $21.7 \mathrm{Merg} \cdot \mathrm{cm}^{-3}$, magnetic energy product up to $2.60 \mathrm{MGOe}$, controllable magnetization from 27.3 to $270.5 \mathrm{emu} \cdot \mathrm{cm}^{-3}$ [29]. These films are expected to be multifunctional and cost-effective alternative for perpendicular magnetic recording bits and a variety of novel devices with high magnetic-noise resistance and thermal stability.

Previous experimental results have shown that the Mn-Ga alloys grown on substrate or in superlattices have affected the tetragonality of the crystal structures, which is attributed to the lattice strain, and this has influence on the magnetic properties and the magnetocrystalline anisotropy of the alloys [18,30-36]. It was found that the crystalline orientation of the Ferromagnetic $\mathrm{L} 10-\mathrm{MnGa}$ thin films, which epitaxially grown on $\mathrm{GaN}$, sapphire, and $\mathrm{MgO}$ substrates, differ due to the influence of the substrate [32]. The in-plane and out-of-plane anisotropy is directly determined by the crystal orientation of the film and could be controlled via selection of the substrates. Moreover, it was reported that the substrate modifies the magnetic properties of $L 1_{0^{-}} \mathrm{Mn}_{x} \mathrm{Ga}(1.2<x<1.5)$ films. Particularly, the $\mathrm{Mn}_{x} \mathrm{Ga}$ film is a hard ferrimagnet when grown on $\mathrm{GaSb}$ (111), becomes a soft ferrimagnet when grown on $\mathrm{Al}_{2} \mathrm{O}_{3}$ (0001), and exhibits an absence of a net magnetic moment when stabilized on a GaSb (100) substrate [33]. These results may be helpful in modifying magnetic anisotropy in thin films for spintronic 
applications. Therefore, understanding the effect of strain in the magnetocrystalline anisotropy and magnetization of $\mathrm{MnGa}$ alloys helps to realize the potential of $\mathrm{Mn}-\mathrm{Ga}$ alloys for use in various applications.

In this work we performed density functional theory (DFT) electronic structure calculations to examine the magnetocrystalline anisotropy energy (MAE) and magnetization of Mn-Ga alloys as function of compositions (Mn and $\mathrm{Ga}$ ) and strain. We investigated the structural and magnetic properties of the alloys as function of compositions and under applied strain. Our calculations show that MAE of Mn-Ga alloys are large. We show that MAE in MnGa and $\mathrm{Mn}_{3} \mathrm{Ga}$ alloys remains large when moderate bi-axial strain is applied. The tensile strain increases MAE in $\mathrm{Mn}_{1.66} \mathrm{Ga}$ alloys. The changes in the electronic structure, specifically the Fermi level position with electron population variation, explain the variation of MAE for different $\mathrm{Mn}$ content. Our study shows that volume expansion of the MnGa alloys increase the energy product of these alloys.

\section{Computational methods}

Self-consistent electronic structure calculations were performed for $\mathrm{MnGa}, \mathrm{Mn}_{3} \mathrm{Ga}$ and $\mathrm{Mn}_{1.66} \mathrm{Ga}$ alloys. The calculations were carried out using the Density-functional theory (DFT) method [37,38] as implemented in the Vienna ab initio simulation package VASP [39]. Projector augmented wave PAW pseudopotentials were used [40]. The generalized gradient approximation (GGA) of Perdew-Burke-Ernzerhof (PBE) form [41] is used for the exchange-correlation functional. We used a $16 \times 16 \times 16 k$-points sampling for $\mathrm{MnGa}$ and $12 \times 12 \times 9$ for $\mathrm{Mn}_{3} \mathrm{Ga}$ and $\mathrm{Mn}_{1.66} \mathrm{Ga}$, the Blöchl's tetrahedron integration method was used [42]. We set the plane-wave-cutoff energy to $350 \mathrm{eV}$ and we choose the convergence criteria for energy of $10^{-6} \mathrm{eV}$. Calculations were performed with relaxation of both atomic positions and lattice parameters. We relax the 
atomic positions of all atoms in the unit cells using Hellmann-Faynmann scheme till forces were less than $0.003 \mathrm{eV} / \AA$. We included the spin-orbit interaction in our calculations and employed force theorem to calculate the $\mathrm{MAE}=\mathrm{E}(\|)-\mathrm{E}(\perp)$ as difference of energies of the system with magnetization along and perpendicular to the easy axis[ $23,43,44]$.

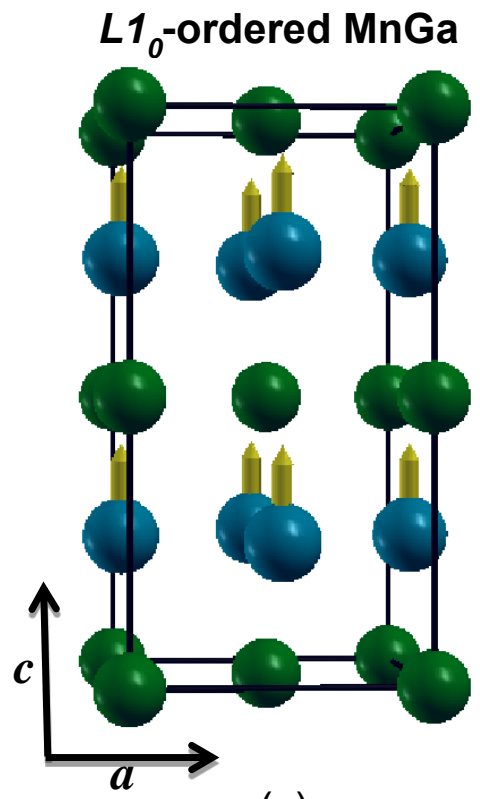

(a)

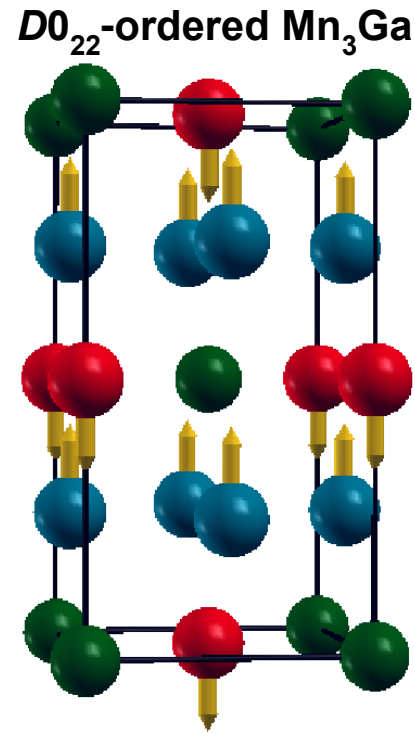

(b)

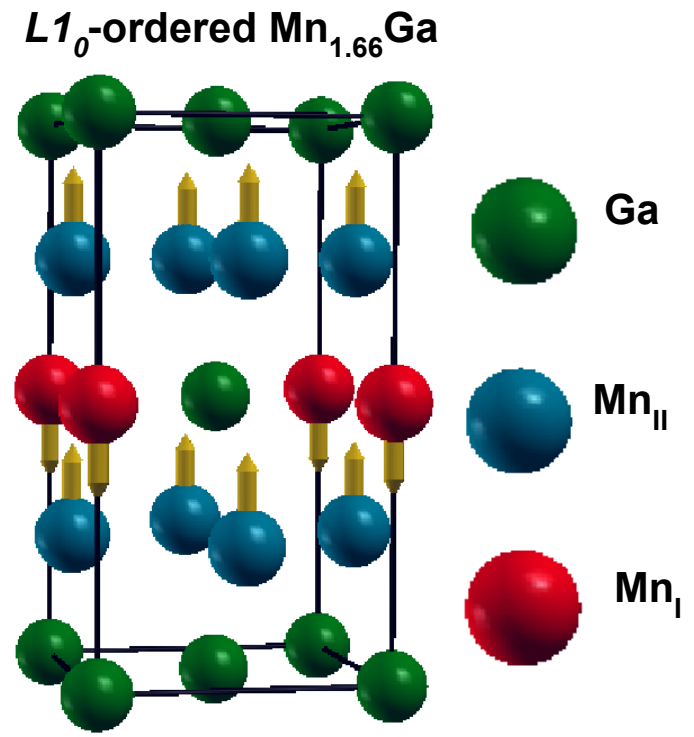

(c)

Figure 1. The unit cell of crystal structure for (a) $\mathrm{Ll}_{0}$-ordered $\mathrm{MnGa}$, (b) $\mathrm{DO}_{22}$-ordered $\mathrm{Mn}_{3} \mathrm{Ga}$, and (c) $L 1_{0}$-ordered $\mathrm{Mn}_{1.66} \mathrm{Ga}$. The unit cell in (a) and (c) is doubled along the $c$ axis for comparison with the $\mathrm{DO}_{22}$ unit cell. In (b), Mn atoms that occupy Wyckoff positions $2 b$ and $4 d$ are denoted by $\mathrm{Mn}_{\mathrm{I}}$ and $\mathrm{Mn}_{\text {II }}$, respectively; Ga atoms are located at the Wyckoff position $2 a$. The $\mathrm{Mn}_{\mathrm{I}}$ atoms in the $D 0_{22}$ structure are replaced by Ga atoms in the $L 1_{0}$ structure. In (c), One $\mathrm{Mn}_{\mathrm{I}}$ atom in the $\mathrm{DO}_{22}$ unit cell is replaced by $\mathrm{Ga}$ atom in the $L 1_{0}$-ordered $\mathrm{Mn}_{1.66} \mathrm{Ga}$ structure. Yellow arrows indicated the electron spin direction.

\section{Results and Discussion}

\subsection{Crystal structures}

Figure 1 shows the unit cells of $L 1_{0}$-ordered $\mathrm{MnGa}, D O_{22}$-ordered $\mathrm{Mn}_{3} \mathrm{Ga}$, and $L 1_{0}$-ordered $\mathrm{Mn}_{1.66} \mathrm{Ga}$ alloys structures adopted in this work to perform systematic electronic structure calculations for pristine and strained structures. The unit cells of $L 1_{0^{-}}$ordered $\mathrm{MnGa}$ and $\mathrm{L} \mathrm{I}_{0^{-}}$ ordered $\mathrm{Mn}_{1.66} \mathrm{Ga}$, in Figure 1 (a) and (c), are doubled along the $c$ axis for accurate quantitative 
comparison of results with the ones of $\mathrm{DO}_{22}$-ordered $\mathrm{Mn}_{3} \mathrm{Ga}$ unit cell. In $\mathrm{DO}_{22}$-ordered $\mathrm{Mn}_{3} \mathrm{Ga}$ unit cell, Figure 1(b), manganese atoms that are located on the base square faces $(1 / 2,1 / 2,0)$ and on the center plane $(0,0,1 / 2)$ are denoted by $\mathrm{Mn}_{\mathrm{I}}$, and manganese atoms, with multiplicity 2 , on the rectangular faces $(0,1 / 2,1 / 4)$ are denoted by $\mathrm{Mn}_{\text {III }}$ Ga atoms are located at the corners and center of the cuboid. The $\mathrm{Mn}_{\mathrm{I}}$ atoms have a tetrahedral nearest neighbor environment. The magnetic moment of $\mathrm{Mn}_{\mathrm{I}}$ is antiparallel to that of $\mathrm{Mn}_{\mathrm{II}}$ in the ground state case. The $\mathrm{Mn}_{\mathrm{I}}$ atoms in the $\mathrm{DO}_{22}$ structure are replaced by $\mathrm{Ga}$ atoms in the $L 1_{0}$-ordered $\mathrm{MnGa}$ unit cell, as shown in Figure 1(a). One Ga atom in the $L 1_{0}$-ordered $\mathrm{MnGa}$ unit cell is replaced by $\mathrm{Mn}_{\mathrm{I}}$ atom in the $L 1_{0^{-}}$ ordered $\mathrm{Mn}_{1.66} \mathrm{Ga}$ unit cell, as shown in Figure 1(c).

Table 1. Lattice Parameters of Mn-Ga alloys. The deviation of lattice parameters from the respective experimental values are shown in brackets.

\begin{tabular}{|c|c|c|c|c|}
\hline \multirow{2}{*}{ Mn-Ga alloy } & \multicolumn{2}{|c|}{ This work } & \multicolumn{2}{c|}{ Experimental } \\
\cline { 2 - 5 } & $a(\AA)$ & $c(\AA)$ & $a(\AA)$ & $c(\AA)$ \\
\hline $\mathrm{MnGa}$ & $3.889[-0.2 \%]$ & $3.617[-0.2 \%]$ & 3.897 & $3.625[$ ref.28] \\
\hline $\mathrm{Mn}_{1.66} \mathrm{Ga}$ & $3.770[-3.1 \%]$ & $3.619[-0.39 \%]$ & 3.893 & $3.633[$ ref.45] \\
\hline $\mathrm{Mn}_{3} \mathrm{Ga}$ & $3.770[-3.7 \%]$ & $7.160[+0.87 \%]$ & 3.909 & $7.098[$ ref.20] \\
\hline
\end{tabular}

The above crystal structures of MnGa alloys were optimization using the total energy minimization. The equilibrium lattice parameter has been computed by variation of the volume as well as the c/a ratio in the supercells. The optimized equilibrium lattice parameters of Mn-Ga alloys are given in Table 1. The equilibrium structural parameters are in very good agreement with experimental values that shown in Table $1[20,28,45] . L 1_{0^{-}}$MnGa lattice parameters, $a$ and $c$, have small deviation of $-0.2 \%$ from the experimental values. $a$ lattice constant of $\mathrm{DO}_{22^{-}}$ $\mathrm{Mn}_{3} \mathrm{Ga}$ has deviations around $-3.7 \%$, while $c$ lattice constant has deviations around $+0.87 \%$ from the experimental values of the same structure. We compare the calculated lattice parameters of 
$L 1_{0^{-}} \mathrm{Mn}_{1.66} \mathrm{Ga}$ with the experimental lattice parameters of $L 1_{0^{-}} \mathrm{Mn}_{1.6} \mathrm{Ga}$ alloy [45], $a$ and $c$ lattice parameters have deviation of $-3.1 \%$ and $-0.39 \%$ from the experimental values of $L 1_{0^{-}} \mathrm{Mn}_{1.6} \mathrm{Ga}$ alloy. The deviations on the calculated lattice constants are typical for the DFT calculations.

\subsection{Magnetocrystalline anisotropy}

The magnetocrystalline anisotropy energy was calculated for Mn-Ga alloys in our study using force theorem. The MAE values are estimated at equilibrium structural parameters for the unstrained Mn-Ga alloys. The calculated value of MAE is equal 24.4 Merg $/ \mathrm{cm}^{3}$ for $L 1_{0^{-}} \mathrm{MnGa}$, which is consistent with the theoretical value of $26 \mathrm{Merg} / \mathrm{cm}^{3}$ and larger than the reported value around $19 \mathrm{Merg} / \mathrm{cm}^{3}$ for the same structure [20,23]. The estimated value of MAE for $L 1_{0^{-}}$ $\mathrm{Mn}_{1.66} \mathrm{Ga}$ is $22.6 \mathrm{Merg} / \mathrm{cm}^{3}$ which is in very good agreement with the reported experimental value of $21.7 \mathrm{Merg} / \mathrm{cm}^{3}$ in $L 1_{0^{-}} \mathrm{Mn}_{1.5} \mathrm{Ga}$ films [29], while larger than the reported value of 15 Merg/ $\mathrm{cm}^{3}$ in $L 1_{0^{-}} \mathrm{Mn}_{1.54} \mathrm{Ga}$ films on $\mathrm{MgO}[13,46]$. The calculated MAE for ground state structure of $\mathrm{DO}_{22}-\mathrm{Mn}_{3} \mathrm{Ga}$ is $27.3 \mathrm{Merg} / \mathrm{cm}^{3}$, which is in good agreement with the previously estimated value using linear muffin-tin orbitals method [13].

To study the effect of strain in MAE of Mn-Ga alloys, we vary the lattice parameters of the super cells of Mn-Ga structures and mapping the MAE values for different $a$ and $c$ lattice constants around the equilibrium values. Figure 2 shows mapping of the MAE values of $\mathrm{Mn}-\mathrm{Ga}$ alloys with respect to the different lattice parameters to represent the strain effect. As we can see in Figure 2, MAE increases upon decrease in volume in MnGa alloy, while it is decreases in $\mathrm{Mn}_{3} \mathrm{Ga}$ alloy. $\mathrm{MAE}$ in stoichiometric $\mathrm{MnGa}$ or $\mathrm{Mn}_{3} \mathrm{Ga}$ compounds remains large when moderate strains are applied. In special case of bi-axial strain both compressive and tensile does not lead to significant change in MAE, the green lines in Figure 2 represent the bi-axial strain (drawn to 
guide the eye. Assuming Poisson ratio $v$ is $0.3 \Delta c / c=-2 v /(1-v) * \Delta a / a=-0.85 * \Delta a / a$. $)$. While tensile strain increases MAE in $\mathrm{Mn}_{1.66} \mathrm{Ga}$ alloys.
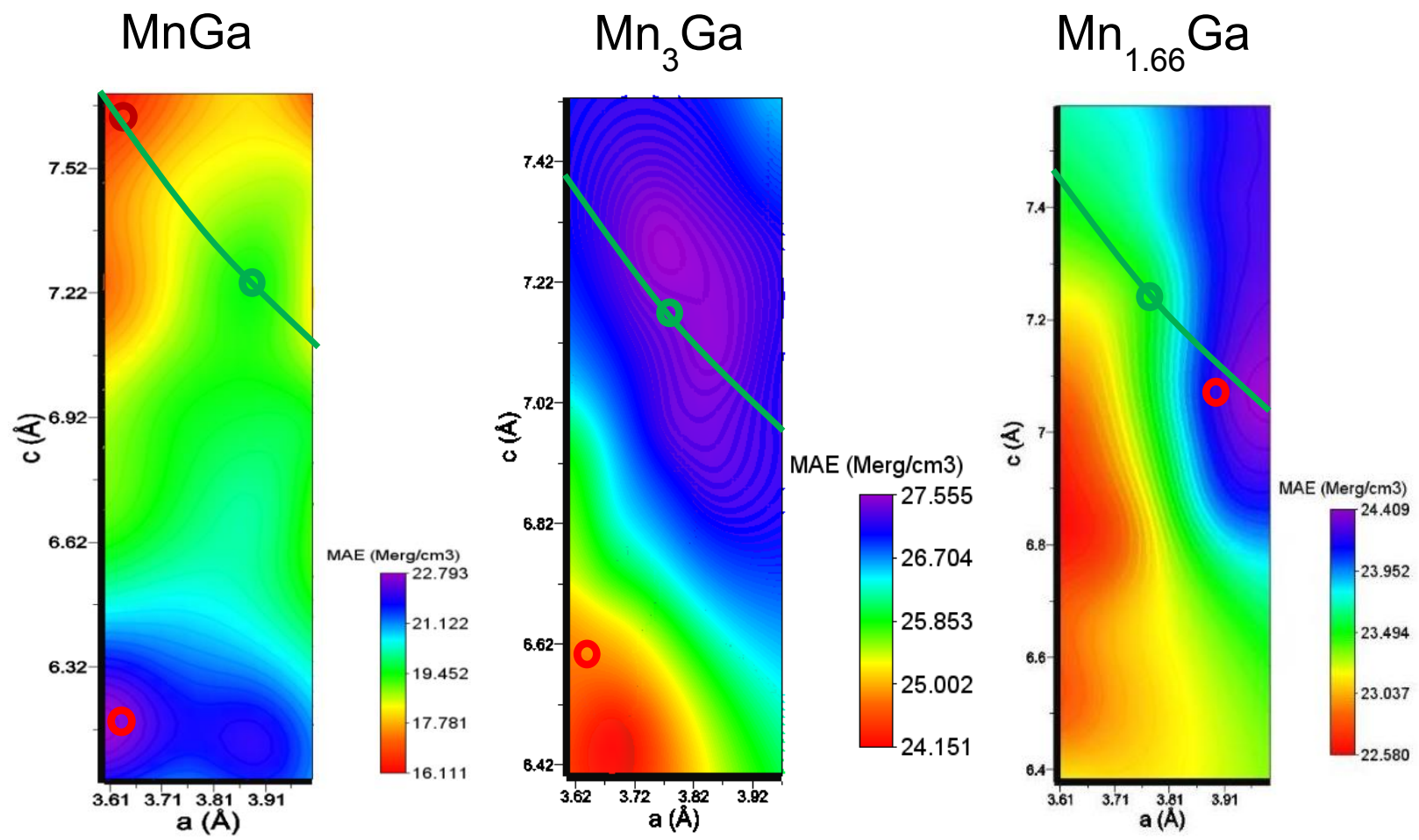

Figure 2. MAE values with respect to the lattice parameters of Mn-Ga alloys. The green circles represent the MAE values at the lowest energy structures of Mn-Ga alloys.

\subsection{Band structures}

To study the effect of strain in the electronic structures of Mn-Ga alloys, we calculated the electronic band-structures for $L 1_{0^{-}} \mathrm{MnGa}, D O_{22^{-}} \mathrm{Mn}_{3} \mathrm{Ga}$, and $L 1_{0^{-}} \mathrm{Mn}_{1.66} \mathrm{Ga}$ alloys, included the spin-orbit interaction in the calculation. We analyze the electronic band-structures, when the magnetization along and perpendicular to the easy axis, for some cases with different lattice parameters, presented by red circles and the equilibrium case with green circle in Figure 2, to show the relation between the change in the electronic structure and the change in MAE as function of Mn. Figures 3,4 and 5 show the electronic band structures of $\mathrm{Ll}_{0^{-}} \mathrm{MnGa}, \mathrm{DO}_{22^{-}}$ $\mathrm{Mn}_{3} \mathrm{Ga}$, and $L 1_{0^{-}} \mathrm{Mn}_{1.66} \mathrm{Ga}$ alloys, respectively, when the magnetic moments out of plane 
(perpendicular to the easy axis) and when it is in plane (along the easy axis) at different lattice parameters to represent the strain effect.

Spin-orbit interaction is described by Hamiltonian

$H_{s o}=\xi(\vec{L} \cdot \vec{S})$

where $\mathrm{L}$ is orbital moment and $\mathrm{S}$ is a spin moment operators, and $\xi$ is spin-orbit coupling constant. The effect of spin-orbit coupling on the band structure provides a splitting of energy levels according to total angular momentum quantum number $m_{J}$. The shift in the band energy reflects the change in total energy. The spin-orbit contribution to energy varies, depending on the orientation of the quantization axis (determined in experiment by an applied magnetic field). This leads to appearance of easy axis direction where the energy is minimal and hard axis where the energy is maximum. In case of uniaxial anisotropy appearing as second order contribution to the energy when expanded over the small parameter of spin-orbit constant, the energy is proportional to

$H=-K_{u} \cos (\theta)^{2}$

where $K_{u}$ is uniaxial anisotropy constant and $\theta$ is an angle between magnetic moment and an easy axis.

The variation of MAE as function of Mn content is related to the change in electronic structure. Specifically, the increasing Mn content changes the hybridization between Mn-Mn and Mn-Ga neighbors. As a result the dispersion curves of $\mathrm{MnGa}$ changes compared with that of $\mathrm{Mn}_{3} \mathrm{Ga}$. The Fermi level position also changes with electron population variation in Mn-Ga alloys. Comparing band dispersions when magnetization is along easy $c$-axis (out-of-xy-plane) and hard axis (in xy-plane), we clearly see shifts in the bands and, particularly, splitting of bands at points of degeneracy (highlighted in Figures 3-5). The large contribution to MAE can be expected when 
band crossing the Fermi surface shifts when magnetization is along the easy or hard axis. In this case the contribution to one-electron energy drastically shifts because of change in population of the band. Surprisingly, although the band structure in three considered MnGa systems is quite different, particularly, in the vicinity of the Fermi level, MAE of all three compounds is quite similar in magnitude. All three show similar features of band energy shift. Because, the spinorbit coupling constant is similar for Mn sites the shift magnitude in three structures is similar as can be seen in Figures 3-5. However, the difference in band structure results in very different response to the applied biaxial strain as can be concluded from Figure 2. For example, the maximum of MAE lays at contracted lattice for both in-plane, $a$, and out-of-plane, $c$, parameters for $\mathrm{MnGa}$, which opposite from $\mathrm{Mn}_{3} \mathrm{Ga}$ where maximum MAE observer at expanded $a$ and $c$. In case of $\mathrm{Mn}_{1.66} \mathrm{Ga} \mathrm{MAE}$ is insensitive to out-of-plane parameter, while increases slightly with the increase of in-plane parameter. 

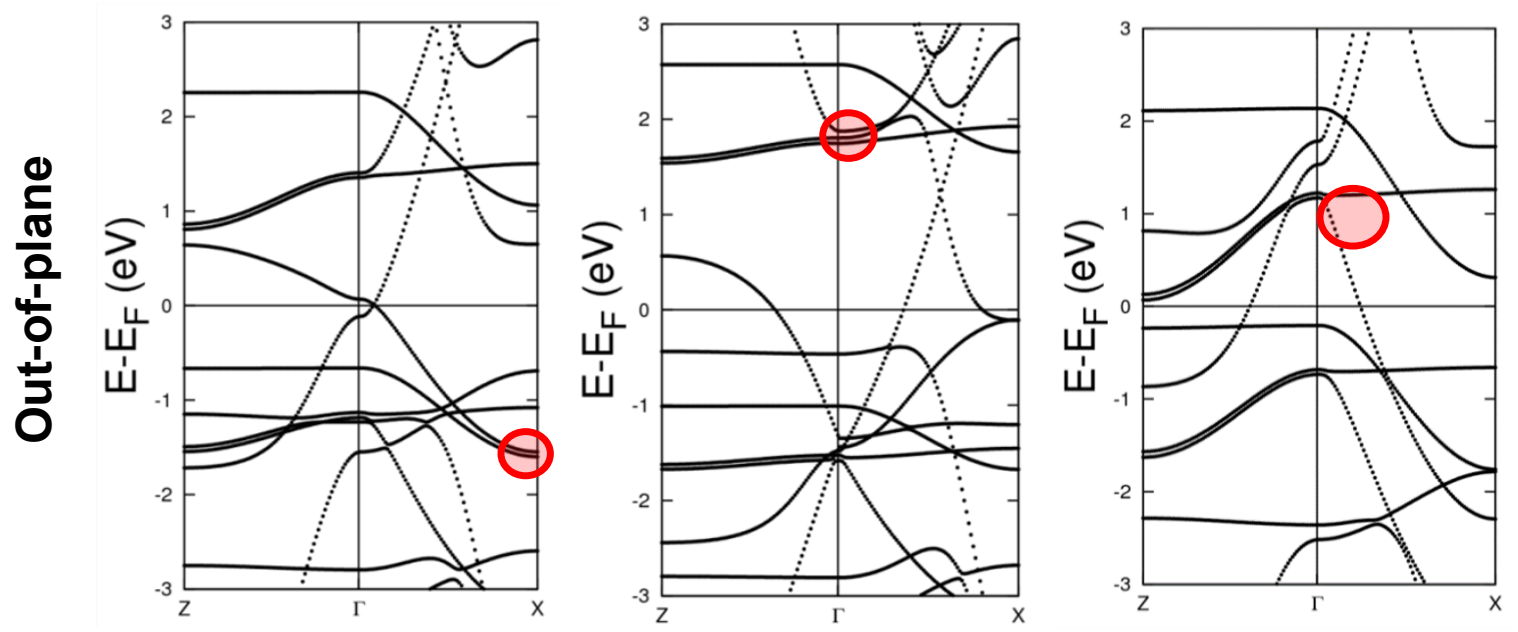

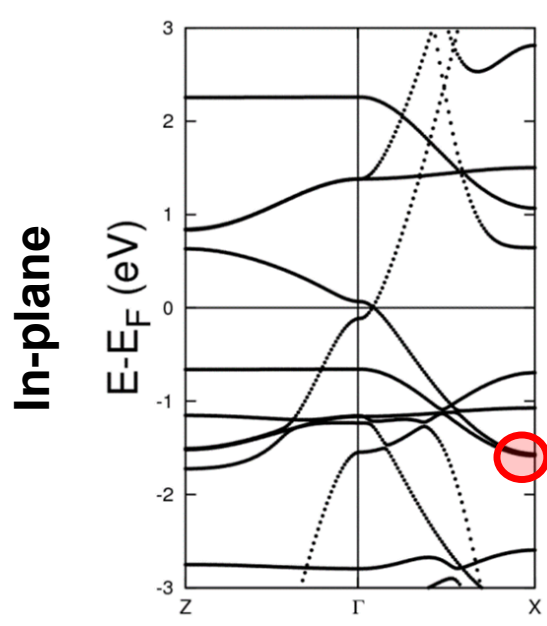

(a)

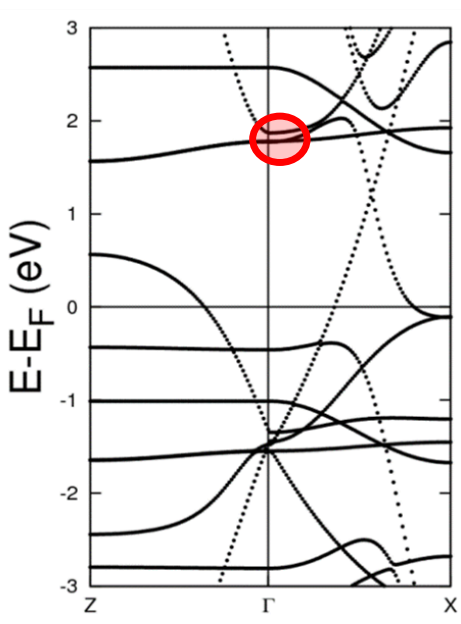

(b)

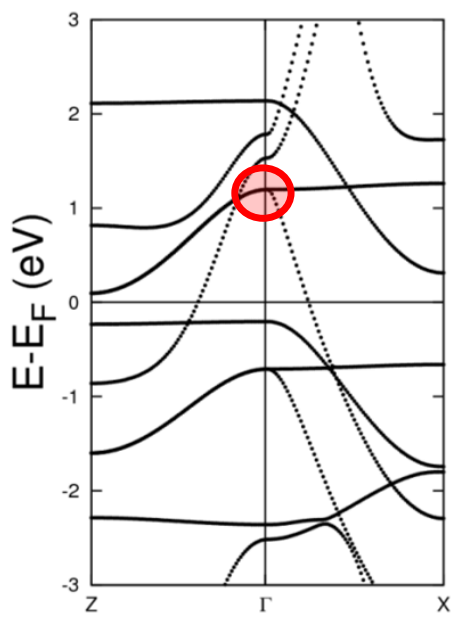

(c)

Figure 3. The electronic band-structures of $L 1_{0^{-}} \mathrm{MnGa}$, when the magnetic moments out of plane (perpendicular to the easy axis) and in plane (along the easy axis) at the following lattice parameters: (a) $a=3.889 \AA, c / a=1.860$ (equilibrium lattice constants), MAE $=24.447 \mathrm{Merg} / \mathrm{cm}^{3}$, (b) $a=3.599 \AA, c / a=$ $2.143, \mathrm{MAE}=12.894 \mathrm{Merg} / \mathrm{cm}^{3}$, and (c) $a=3.599 \AA, c / a=1.719, \mathrm{MAE}=28.1475 \mathrm{Merg} / \mathrm{cm}^{3}$. 

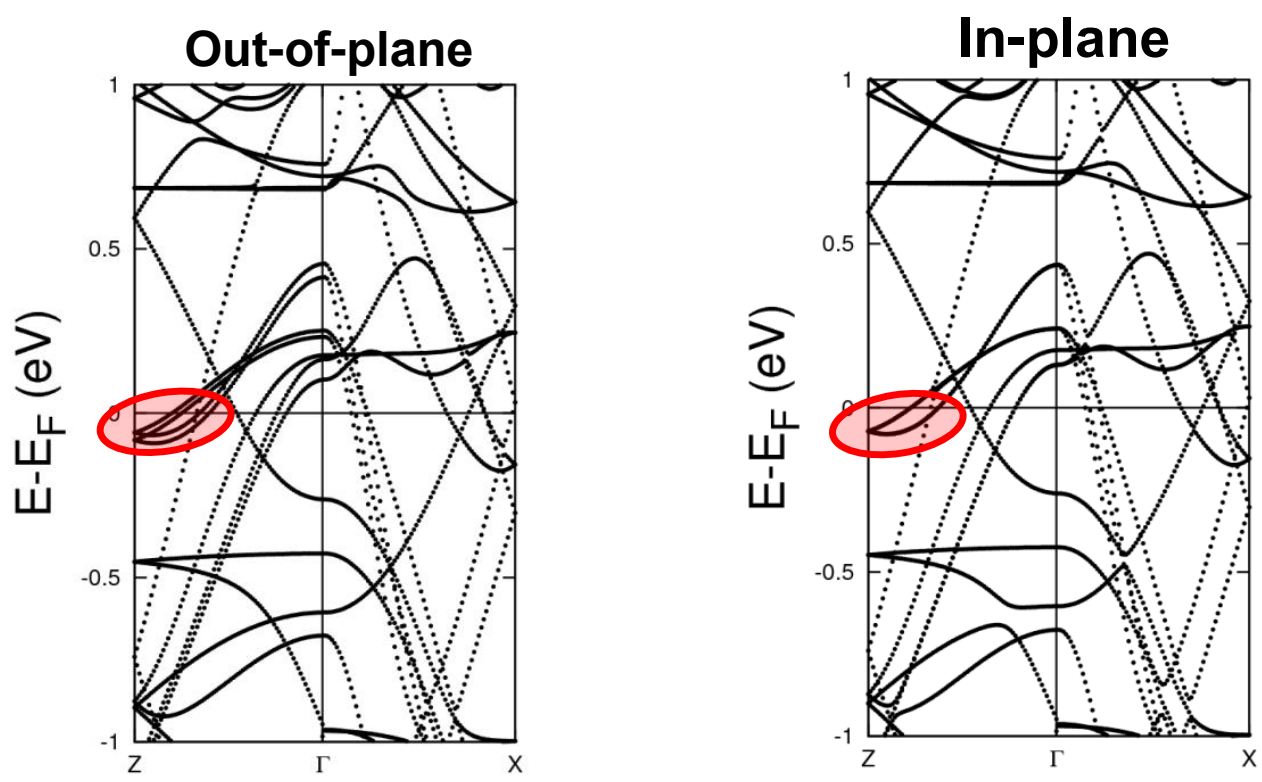

(a) $a=3.77 \AA, c / a=0.9496 M A E=27.3477 \mathrm{Merg} / \mathrm{cm}^{3}$
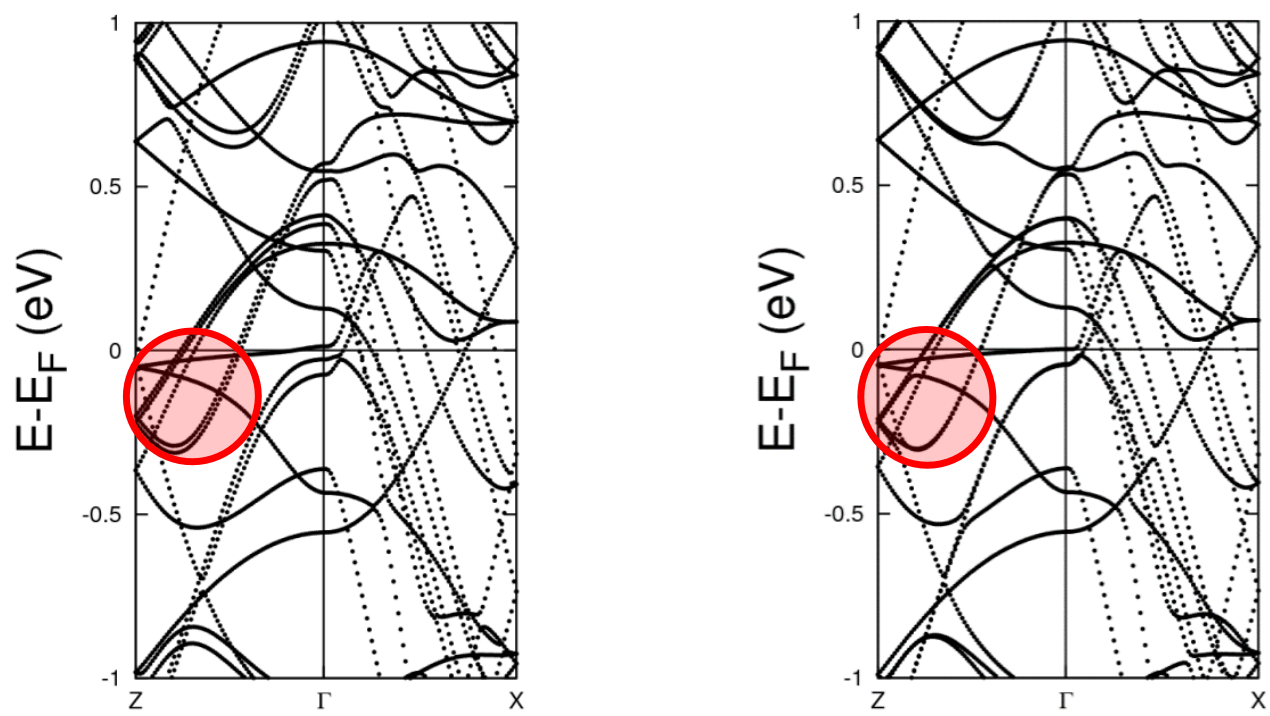

(b) $a=3.604$ A, $c / a=0.92207 M A E=23.8118 \mathrm{Merg} / \mathrm{cm}^{3}$

Figure 4. The electronic band-structures of $\mathrm{DO}_{22}-\mathrm{Mn}_{3} \mathrm{Ga}$, when the magnetic moments out of plane (perpendicular to the easy axis) and in plane (along the easy axis) at the following lattice parameters: (a) $a=3.77 \AA, c / a=0.9496$ (equilibrium lattice constants), and (b) $a=3.604 \AA, c / a=$ 0.92207. MAE values are shown in the figure for comparison. 

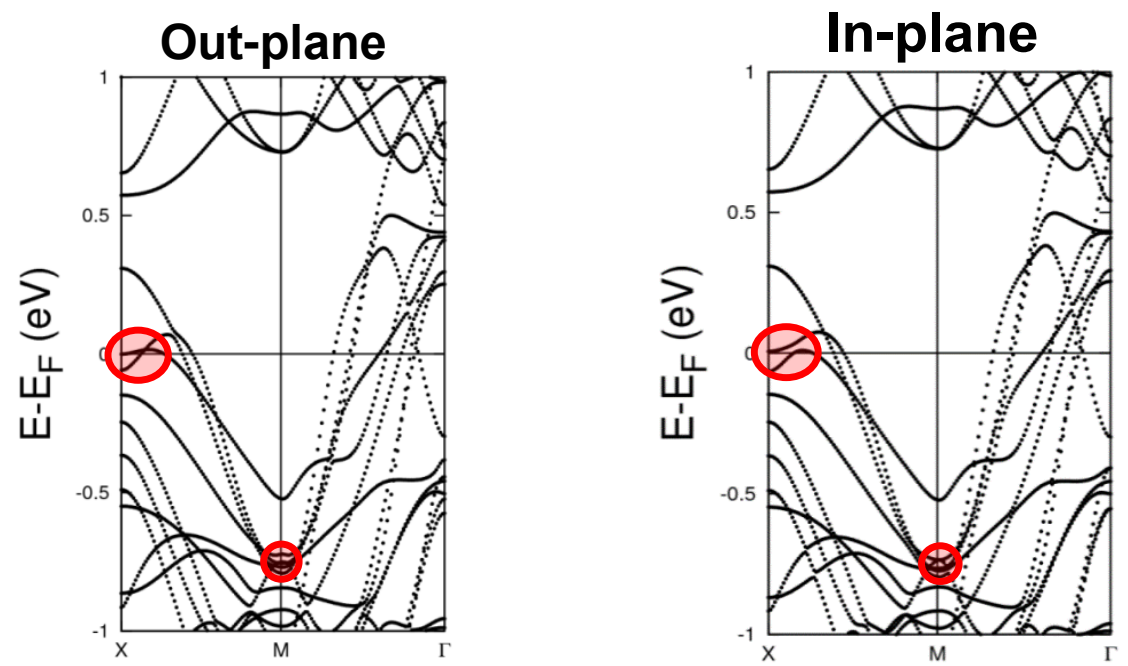

(a) $\mathrm{a}=3.77 \AA, \mathrm{c} / \mathrm{a}=0.95996, \mathrm{MAE}=22.583 \mathrm{Merg} / \mathrm{cm}^{3}$
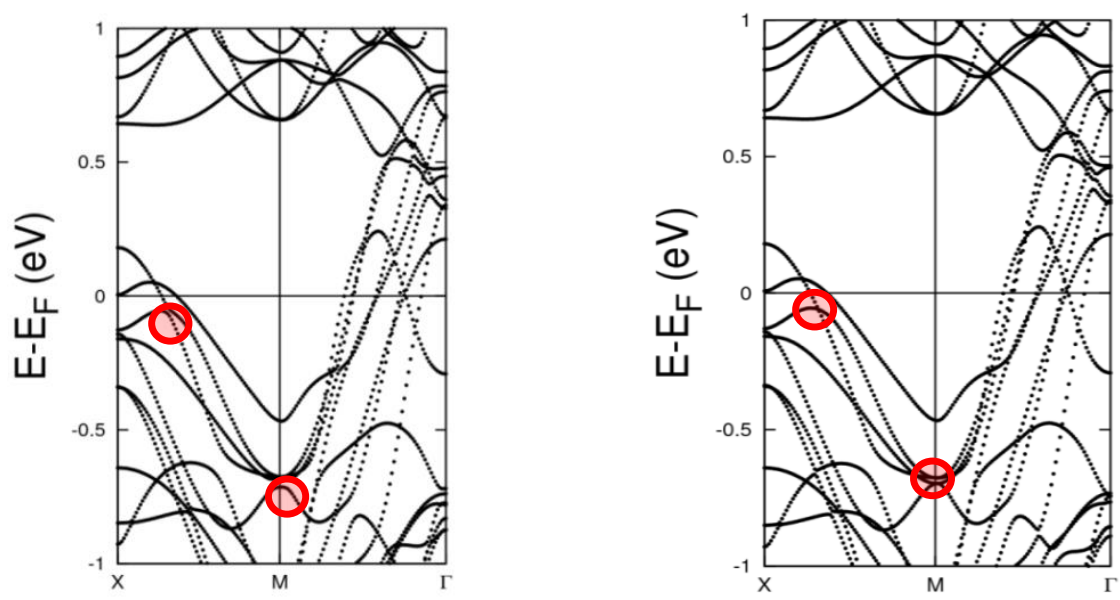

(b) $\mathrm{a}=3.904 \AA$, $\mathrm{c} / \mathrm{a}=0.905, \mathrm{MAE}=25.336 \mathrm{Merg} / \mathrm{cm}^{3}$

Figure 5. The electronic band-structures of $L 1_{0^{-}} \mathrm{Mn}_{1.66} \mathrm{Ga}$, when the magnetic moments out of plane (perpendicular to the easy axis) and in plane (along the easy axis) at the following lattice parameters: (a) $a=3.77 \AA, c / a=0.95996$ (equilibrium lattice constants), and (b) $2-a=3.904 \AA$, $c / a=0.905$. MAE values are shown in the figure for comparison.

\subsection{Magnetization and energy product of Mn-Ga alloys}

The net magnetic moments per unit cell in the ground state are $9.84 \mu_{\mathrm{B}}$ for $L 1_{0}$-ordered $\mathrm{MnGa}, 3.52 \mu_{\mathrm{B}}$ for $D 0_{22}$-ordered $\mathrm{Mn}_{3} \mathrm{Ga}$, and $6.52 \mu_{\mathrm{B}}$ for $L 1_{0}$-ordered $\mathrm{Mn}_{1.66} \mathrm{Ga}$. For $L 1_{0}$-ordered $\mathrm{Mn}_{1.66} \mathrm{Ga}$, the extra $\mathrm{Mn}$ atoms replace the Ga atoms (Figure 1(c)). The local magnetic moment for Mn atom substituting for Ga couples antiferromagnetically to the magnetic moments of the $\mathrm{Mn}_{I I}$ atoms, and this coupling reduces the net magnetic moment of the $\mathrm{Mn}-\mathrm{Ga}$ alloy from $9.84 \mu_{\mathrm{B}}$ 
for $L 1_{0}$-ordered $\mathrm{MnGa}$ to $6.52 \mu_{\mathrm{B}}$ for $L 1_{0}$-ordered $\mathrm{Mn}_{1.66} \mathrm{Ga}$. The ground state structure of $D 0_{22}{ }^{-}$ ordered $\mathrm{Mn}_{3} \mathrm{Ga}$ exhibits ferrimagnetic order, where the magnetic moment of $\mathrm{Mn}_{\mathrm{I}}$ is antiparallel to the magnetic moment of $\mathrm{Mn}_{\mathrm{II}}$, as shown in Figure 1 (b). The total magnetic moment of $1.76 \mu_{\mathrm{B}}$ in the primitive $\mathrm{DO}_{22}$ cell of $\mathrm{Mn}_{3} \mathrm{Ga}$ is low due to the partial compensation of the moments of the $\mathrm{Mn}$ atoms on the two different sites. This magnetization value of $\mathrm{Mn}_{3} \mathrm{Ga}$ in $D 0_{22}$ structure agrees well with previously reported values that calculated using pseudopotential and full potential DFT calculations $[19,20]$.

The local magnetic moment of the $\mathrm{Mn}$ atoms at $\mathrm{Mn}_{\text {II }}$ sites in $D 0_{22} \mathrm{Mn}_{3} \mathrm{Ga}$ and $L 1_{0}$-ordered $\mathrm{Mn}_{1.66} \mathrm{Ga}$ are not largely different from that of $L 1_{0^{-}} \mathrm{MnGa}$. In fact, the magnetic moments of $\mathrm{Mn}$ atoms at the $\mathrm{Mn}_{\mathrm{II}}$ sites in $\mathrm{DO}_{22} \mathrm{Mn}_{3} \mathrm{Ga}$ and $L 1_{0} \mathrm{Mn}_{1.66} \mathrm{Ga}$ are estimated as $2.35 \mu \mathrm{B}$ and $2.43 \mu \mathrm{B}$, respectively. These values are close to that for $L 1_{0^{-}} \mathrm{MnGa}(2.58 \mu \mathrm{B})$. The magnetic moment for $\mathrm{Mn}_{\mathrm{I}}$ is estimated to be $-2.88 \mu \mathrm{B}$ in case of $\mathrm{DO}_{22} \mathrm{Mn}_{3} \mathrm{Ga}$ and $-2.94 \mu \mathrm{B}$ for $L 1_{0}$-ordered $\mathrm{Mn}_{1.66} \mathrm{Ga}$. The calculated values for local magnetic moments of Mn-Ga alloys are in agreement with the previously experimental and theoretical obtained values for $D 0_{22} \mathrm{Mn}_{3} \mathrm{Ga}$ and $L 1_{0} \mathrm{MnGa}$ alloys $[19,20,23,28,46]$. The total orbital magnetic moments and the atomic orbital magnetic moments of Mn_Ga alloys are shown in Table 2. The orbital moments are parallel to the magnetic moments. The magnitude of orbital moments is of the order of $0.03 \mu_{\mathrm{B}}$. This value is not very large taking into account substantial MAE. This is due to a partial quenching of orbital moment which is observed in systems with cubic or tetragonal symmetry (of small tetragonality $c / a$ ). 
Table2. The total orbital magnetic moments and the atomic orbital magnetic moments (in $\mu_{\mathrm{B}}$ ) of Mn_Ga alloys.

\begin{tabular}{|l|c|c|c|c|c|c|}
\hline & \multicolumn{2}{|c|}{ MnGa } & \multicolumn{2}{c|}{$\mathrm{Mn}_{3} \mathrm{Ga}$} & \multicolumn{2}{c|}{$\mathrm{Mn}_{1.66} \mathrm{Ga}$} \\
\hline & Out-plane & In-plane & Out-plane & In-plane & Out-plane & In-plane \\
\hline $\mathrm{Mn}_{\text {II }}$ & 0.034 & 0.035 & 0.028 & 0.023 & 0.029 & $0.026,0.029$ \\
\hline $\mathrm{Mn}_{\mathrm{I}}$ & & & -0.025 & -0.019 & -0.018 & -0.018 \\
\hline $\mathrm{Ga}$ & -0.001 & 0.001 & -0.001 & 0.00 & -0.001 & 0.00 \\
\hline Total & 0.03 & 0.04 & 0.06 & 0.05 & 0.10 & 0.09 \\
\hline
\end{tabular}

The energy product of a magnet is governed by its magnetization, $M$. In fact, the maximum energy product is $1 / 4 \mu_{B} M^{2}$. The calculated theoretical limits of energy product of $\mathrm{MnGa}, \mathrm{Mn}_{3} \mathrm{Ga}$ and $\mathrm{Mn}_{1.66} \mathrm{Ga}$ are 23.65, 4.06 and 13.64 MGOe, respectively. As we can see MnGa has a very large theoretical limit of energy product. However, it suffers from the low coercivity near or above room temperature. $\mathrm{Mn}_{3} \mathrm{Ga}$ has a small energy product due to low magnetization, reflecting ferromagnetic coupling in this system. $\mathrm{Mn}_{1.66} \mathrm{Ga}$ shows magnetization reduced compared to $\mathrm{MnGa}$, but shows large coercivity in recent experimental studies. ${ }^{32}$ Thus, finding a way to increase magnetization can assist in improving an energy product of the magnet. We explore the effect of the strain on the magnetization. This question is related to the wellknown magnetovolume effect in magnetism of 3d-metals [47]. Typically, the volume increase results in the increased magnetization, but the details of the increase are strongly dependent on the specific system $[47,48]$. To study the effect of strain in the total magnetic moments of Mn-Ga alloys, we map the total magnetic moments the for $L 1_{0^{-}} \mathrm{MnGa}, D O_{22^{-}} \mathrm{Mn}_{3} \mathrm{Ga}$, and $L 1_{0^{-}} \mathrm{Mn}_{1.66} \mathrm{Ga}$ alloys as function of lattice parameters as shown in Figure 6. As we can see in the figure, total magnetization increases upon increases in both in in-plane axis $a$ or easy axis $c$, in all $\mathrm{Mn}-\mathrm{Ga}$ alloys as expected from magnetovolume effect picture. Thus, increase of magnetization should 
be achieved by alternative methods such as doping or forming an exchange spring composite with high magnetization soft phase [49].
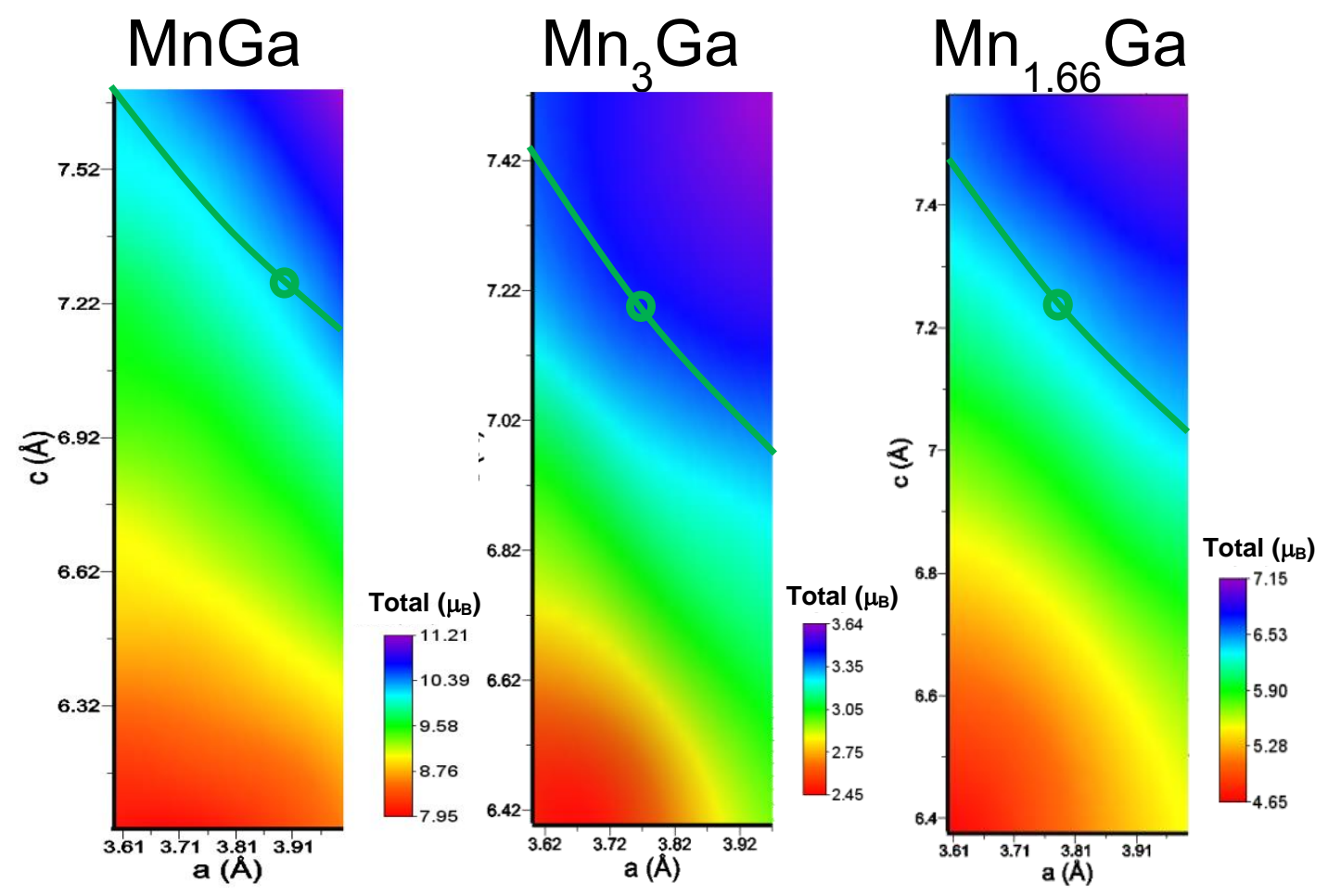

Figure 6. The total magnetic moments ( $\mu_{\mathrm{B}}$ ) of (a) $\mathrm{L1}_{0^{-}} \mathrm{MnGa}$, (b) $\mathrm{DO}_{22^{-}} \mathrm{Mn}_{3} \mathrm{Ga}$, and (c) $\mathrm{L1}_{0^{-}}$ $\mathrm{Mn}_{1.66} \mathrm{Ga}$ alloys with different lattice parameters. The green lines in the figure represent the biaxial strain, and the green circular represent the lower energy structure.

\section{Conclusions}

In our study, we investigated the structural and magnetic properties of the Mn-Ga alloys as function of composition of $\mathrm{Mn}$ and Ga under applied strain. We examine the variation of the magnetocrystalline anisotropy (MAE) and magnetization of Mn-Ga alloys as function of strain using DFT calculations. While MAE is very large in three considered alloys, e.g. $\mathrm{MnGa}, \mathrm{Mn}_{3} \mathrm{Ga}$ and $\mathrm{Mn}_{1.66} \mathrm{Ga}$, MAE can be further increased by increasing the volume in MnGa alloy, and by increasing the volume in $\mathrm{Mn}_{3} \mathrm{Ga}$ alloy. MAE is very robust with respect to the application of moderate strain both compressive and tensile. Tensile strain increases MAE in $\mathrm{Mn}_{1.66} \mathrm{Ga}$ alloys. 
The variation of MAE as function of Mn content is related to the change in electronic structure as function of Mn content. We find that magnetic moment is relatively insensitive to moderate tensile bi-axial strains to MnGa alloys. However, the theoretical limit of energy product could be increased in the MnGa alloys by expanding the volume using doping or strain engineering in composite materials.

\section{Acknowledgment}

This work was supported by AFOSR and NSF (Grant No. DMR-1310542). The University of Nebraska Holland Computing Center has provided computations resources.

\section{References}

[1] A. T. McCallum, P. Krone, F. Springer, C. Brombacher, M. Albrecht, E. Dobisz, M. Grobis, D. Weller, O. Hellwig, Appl. Phys. Lett. 98 (2011) 242503.

[2] W. A. Challener, C. Peng, A. V. Itagi, D. Karns, W. Peng, Y. Peng, X. M. Yang, X. Zhu, N. J. Gokemeijer, Y. T. Hsia, G. Ju, R. E. Rottmayer, M. A. Seigler, E. C. Gage, Nat. Photon. 3 (2009) 220-224.

[3] M. Weisheit, S. Fähler, A. Marty, Y. Souche, C. Poinsignon, D. Givord, Science 315 (2007) 349-351.

[4] T. Seki, M. Kohda, J. Nitta, K. Takanashi, Appl. Phys. Lett. 98 (2011) 212505.

[5] I. M. Miron, K. Garello, G. Gaudin, P. J. Zermatten, M. V. Costachel, S. Auffret, S. Bandiera, B. Rodmacq, A. Schuhl, P. Gambardella, Nature 476 (2011) 189-193.

[6] C. L. Zha, R. K Dumas, J. W. Lau, S. M. Mohseni, S. R. Sani, I. V. Golosovsky, A. F. Monsen, J. Nogues, J. Akerman, J. Appl. Phys. 110 (2011) 093902-4.

[7] T. J. Nummy, S. P. Bennett, T. Cardinal, D. Heiman, Appl. Phys. Lett. 99 (2011) 252506.

[8] O. Gutfleisch, M. A. Willard, E. Brück, C. H. Chen, S. G. Sankar, J. P. Liu, Adv. Mater. 23 (2011) 821-842. 
[9] S. Mangin, D. Ravelosona, J. A. Katine, M. J. Carey, B. D. Terris, E. Fullerton, Nat. Mater. 5 (2006) 210-215.

[10] S. Ikeda, K. Miura, H. Yamamoto, K. Mizunuma, H. D. Gan, M. Endo, S. Kanai, J. Hayakawa, F. Matsukura, H. Ohno, Nat. Mater. 9 (2010) 721-724.

[11] S. S. P. Parkin, C. Kaiser, A. Panchula, P. M. Rice, B. Hughes, M. Samant, S. H. Yang, Nature Mater. 3 (2004) 862-867.

[12] E. Lu, D. C. Ingram, A. R. Smith, J. W. Knepper, F. Y. Yang, Phys. Rev. Lett. 97 (2006) 146101-4.

[13] S. Mizukami, F. Wu, A. Sakuma, J. Walowski, D. Watanabe, T. Kubota, X. Zhang, H. Naganuma, M. Oogane, Y. Ando, T. Miyazaki, Phys. Rev. Lett. 106 (2011) 117201-4.

[14] C. Adelmann, J. L. Hilton, B. D. Schultz, S. McKernan, C. J. Palmstrøm, X. Lou, H. S. Chiang, P. A. Crowell, Appl. Phys. Lett. 89 (2006) 112511-4.

[15] F. Wu, E. P. Sajitha, S. Mizukami, D. Watanabe, T. Miyazaki, H. Naganuma, M. Oogane, Y. Ando, Appl. Phys. Lett. 96 (2010) 042505.

[16] G. Kim, Y. Sakuraba, M. Oogane, Y. Ando, T. Miyazaki, Appl. Phys. Lett. 92 (2008) 172502.

[17] K. M. Krishnan, Appl. Phys. Lett. 61 (1992) 2365.

[18] M. Tanaka, J. P. Harbison, J. Deboeck, T. Sands, B. Philips, T. L. Cheeks, V. G. Keramidas, Appl. Phys. Lett. 62 (1993)1565.

[19] B. Balke, G. H. Fecher, J. Winterlik, C. Felser, Appl. Phys. Lett. 90 (2007)152504.

[20] J. Winterlik, B. Balke, G. H. Fecher, C. Felser, Phys. Rev. B 77 (2008) 054406-054417.

[21] F. Wu, S. Mizukami, D. Watanabe, H. Naganuma, M. Oogane, Y. Ando, T. Miyazaki, Appl. Phys. Lett. 94 (2009)122503-4. 
[22] H. Kurt, K. Rode, M. Venkatesan, P. Stamenov, J. M. D. Coey, Phys. Rev. B 83 (2011) 020405(R)- 020408(R).

[23] A. Sakuma, J. Magn. Magn. Mater. 187 (1998)105-112.

[24] Z. Yang, J. Li, D. Wang, K. Zhang, X. Xie, J. Magn. Magn. Mater. 182 (1998) 369-374.

[25] L. J. Zhu, J. H. Zhao, Appl. Phys. A 111 (2013) 379-38.

[26] T. A. Bither, W. H. Cloud, J. Appl. Phys. 36 (1965)1501.

[27] E. Krén, G. Kádár, Solid State Commun. 8 (1970) 1653-655.

[28] H. Niida, T. Hori, H. Onodera, Y. Yamaguchi, Y. Nakagawa, J. Appl. Phys. 79 (1996) 5946.

[29] L. Zhu, S. Nie, K. Meng, D. Pan, J. Zhao, H. Zheng, Adv. Mater. 24 (2012) 4547-455.

[30] M. Tanaka, J. P. Harbison, T. Sands, B. Philips, T. L. Cheeks, J. Deboeck, L. T. Florez, V. G. Keramidas, Appl. Phys. Lett. 63 (1993) 696.

[31] M. Tanaka, Mater. Sci. Eng. B 31 (1995) 117-125.

[32] K. Wang, E. Lu, J. W Knepper, F. Yang, A. R. Smith, Appl. Phys. Lett. 98 (2011)162507.

[33] W. Feng, D. V. Thiet, D. D. Dung, Y. Shin, S. Chob, J. Appl. Phys. 108 (2010) 113903.

[34] C. L. Zha, R. K. Dumas, J. W. Lau, S. M. Mohseni, S. R. Sani, I. V. Golosovsky, A. F. Monsen, J. Nogues, J. Akerman, J. Appl. Phys. 110 (2011) 093902.

[35] A. Bedoya-Pinto, C. Zube, J. Malindretos, A. Urban, A. Rizzi, Phys. Rev. B 84 (2011)104424.

[36] H. Kurt, K. Rode, M. Venkatesan, P. Stamenov, J. M. D. Coey, Phys. Status Solidi B 248 (2011) 2338-2344.

[37] P. Hohenberg, W. Kohn, Phys. Rev. 136 (1964) B864-B871. 
[38] W. Kohn, L. J. Sham, Phys. Rev. 140 (1965) A1133- A1138.

[39] G. Kresse, D. Joubert, Phys. Rev. B 59 (1999) 1758-1775.

[40] P. E. Blöchl, Phys. Rev. B 5017953 (1994).

[41] J. P. Perdew, K. Burke, M. Ernzerhof, Phys. Rev. Let. 77 (1996) 3865-3879.

[42] P. E. Blöchl, O. Jepsen, O. K. Andersen, Phys. Rev. B 49 (1994) 16223-16233.

[43] D. Hobbs, G. Kresse, J. Hafner, Phys. Rev. B. 62 (2000) 11556-11570.

[44] J. Hafner, Computer Physics Communications 177 (2007) 6-13.

[45] Y. Huh, P. Kharel, V. R. Shah, X. Z. Li, R. Skomski, D. Sellmyer, J. Appl. Phys. 114 (2013) 013906.

[46] S. Mizukami, T. Kubota, F. Wu, X. Zhang, T. Miyazaki, H. Naganuma, M. Oogane, A. Sakuma, Y. Ando, Phys. Rev. B. 85 (2012) 014416-014421.

[47] V. L. Moruzzi, P. M. Marcus, J. Kübler, Phys. Rev. B 39 (1989) 6957.

[48] R. Sabiryanov, O. Mryasov, S. Bose, Phys. Rev. B 51 (1995) 8958.

[49] E. F. Kneller, R. Hawig, IEEE Transactions on Magnetics 27 (1991) 3588-3600. 